\title{
BIBECHANA
}

\section{Some results on asymptotically normable Frechet spaces}

\author{
G.K. Palei ${ }^{\mathbf{a}}$, N.P. Sah ${ }^{\mathbf{*} *}$ \\ ${ }^{a}$ Department of Mathematics, B.N. College, Patna University, Patna, India \\ ${ }^{\mathrm{b}}$ Department of Mathematics, M.M.A.M. Campus (Tribhuvan University), Biratnagar, Nepal \\ Article history: Received 8 October 2010; Revised 18 November 2010; Accepted 20 November 2010
}

\begin{abstract}
.
In this paper, it is shown that the asymptotically normable spaces are the smallest class of Frechet spaces which contains the nuclear Kothe spaces with continuous norm, the Banach spaces and is closed under $\varepsilon$-tensor products and sub-spaces. Again our main aim will be to construct an example of a Kothe space which is Montel, admits a continuous norm, but still is not asymptotically normable.
\end{abstract}

Keywords: Asymptotically normable; Frechet space; Kothe space

\section{Introduction}

Let $\mathrm{E}$ be a Frechet space, \|\|$_{1} \leq\|\|_{2} \leq \ldots .$. a fundamental system of seminorms on $\mathrm{E}$ and $\mathrm{U}_{\mathrm{k}}=\left\{\mathrm{x} \in \mathrm{E}:\|\mathrm{x}\|_{\mathrm{k}} \leq 1\right\}$ for every $\mathrm{k}$. E is called asymptotically normable, if there is a $\mathrm{k}_{\mathrm{o}}$ such that for every $\mathrm{k} \geq \mathrm{k}_{\mathrm{o}}$ there is a $\mathrm{p}$ so that the seminorms \|\|$_{k_{o}}$ and \|\|$_{k}$ define equivalent topologies on $\mathrm{U}_{\mathrm{p}}$. It is easy to see that in this case \|\|$_{k_{o}}$ is in fact a norm.

This class of spaces appears in investigations about the structure of Frechet spaces and about the behaviour of their operators as a natural counterpart of the class of quasi-normable spaces introduced by Grothendieck [1]. While the quasi-normable frechet spaces E are those which admit an $\Omega$-type condition [2] and for which there exists a nontrivial Frechet space $F$ with $\operatorname{Ext}^{1}(\mathrm{~F}, \mathrm{E})=0$ [2], the asymptotically normable Frechet spaces $\mathrm{E}$ are those which admit a DNtype condition [3] and for which thee exists a nontrivial Frechet space $\mathrm{F}$ with $\operatorname{Ext}^{1}(\mathrm{E}, \mathrm{F})=0$. Nontrivial here could mean: an infinite dimensional nuclear Kothe space.

In [2] it is shown that the quasi-normable spaces are the quotient spaces of standard spaces of the form

$$
\lambda(A, E)=\left\{x=\left(x_{j}\right)_{j} \in E^{N}:\|x\|_{k}=\sum_{j}\left\|x_{j}\right\|_{k} a_{j, k}<+\infty \text { for all } k\right\}
$$

where $\mathrm{E}$ is a Banach space and $\mathrm{A}=\left(\mathrm{a}_{\mathrm{j}, \mathrm{k}}\right)$ a matrix with $\mathrm{O}<\mathrm{a}_{\mathrm{j}, \mathrm{k}} \leq \mathrm{a}_{\mathrm{j}, \mathrm{k}+1}$ for all $\mathrm{k}$ and

\footnotetext{
* Corresponding author: Nagendra Pd. Sah, Department of Mathematics, M.M.A.M. Campus(Tribhuvan University), Biratnagar, Nepal
} 


$$
\sum_{j} \frac{a_{j, k}}{a_{j, k+1}}<+\infty
$$

We can show that the asymptotically normable spaces are the subspaces of these standard spaces. They are the smallest class of Frechet spaces which contains the nuclear Kothe spaces with continuous norm, the Banach spaces and is closed under $\varepsilon$-tensor products and subspaces.

A. We use the standard terminology of the theory of locally convex spaces as in [4]. For the theory of Ext \& the splitting conditions we refer to [3] \& [5]. By ( \|\|$\left._{k}\right)$ we always denote an increasing sequence of seminorms defining the topology of a Frechet space $E$ and $U_{k}=\{x \in E$ : $\left.\|\mathrm{x}\|_{\mathrm{k}} \leq 1\right\}$. In this section we collect some simple results in order to see the concept of asymptotic normability in a proper perspective.

Let $\varphi:(0, \infty) \rightarrow\left(0, \infty\right.$, be a strictly increasing function. We say $E$ satisfies $\left(\mathrm{DN}_{\varphi}\right)$ if we have a $\mathrm{k}_{\mathrm{o}}$ with the property that for every $\mathrm{k}$ there is a $\mathrm{p}$ and $\mathrm{C}>0$ so that the inequality

$$
\|\mathrm{x}\|_{\mathrm{k}} \leq \mathrm{C} \varphi(\gamma)\|x\|_{k_{o}}+\frac{1}{\gamma}\|\mathrm{x}\|_{\mathrm{p}}
$$

holds for every $\mathrm{x} \in \mathrm{E}$ and $\gamma>0$ [3]. In the case $\varphi(\gamma)=\gamma$ this condition, denoted by (DN), was introduced by D. VOG T in [3] to characterize initially the subspaces of the space (s) of rapidly decreasing sequences. Subsequently it was proved by him in [5] that every space of type (DN) is isomorphic to a subspace of $\mathrm{L}_{\infty}(\mathrm{I}) \otimes_{\pi}$ (s) for some index set $\mathrm{I}$.

Consider also the following condition: there is a $k_{o}$ such that for every $k \geq k_{o}$ we have $p$ $\geq \mathrm{k}_{\mathrm{o}}$ with the following property: every sequence in $\mathrm{E}$ which is Cauchy with respect to \|\|$_{\mathrm{p}}$ and converges to 0 with respect to \|\|$_{k_{o}}$ even converges to 0 with respect to \|\|$_{\mathrm{k}}$.

Proposition 1.1 Every asymptotically normable Frechet space is countably normable.

We can obtain the proof from [1], page 176.

Proposition 1.2 The following are equivalent for a Frechet space E :

(1) E is asymptotically normable.

(2) There is $k_{o}$ such that for every $k$ there is a p such that for every $\varepsilon>0$ we can choose $M>0$ with \|\|$_{\mathrm{k}} \leq \mathrm{M}\|\|_{k_{o}}+\varepsilon\|\|_{\mathrm{p}}$.

(3) E has property $\left(\mathrm{DN}_{\varphi}\right)$ for some $\varphi$.

Proposition 1.3 A Frechet-Schwartz space is asymptotically normable if and only if it is countably normable.

The question whether these classes coincide in general we shall answer in the negative in the next section.

Before we do this let us recall that a Frechet space E is called locally normable [6] if there is a $\mathrm{k}_{\mathrm{o}}$ such that on every bounded set $\mathrm{B}$ the topologies induced by $\mathrm{E}$ and by \|\|$_{k_{o}}$ coincide. Obviously every asymptotically normable space is locally normable. On the other hand every Frechet-Montel space admitting a continuous norm is locally normable. Hence, in view of Proposition 1.3, every Frechet-Schwartz space admitting a continuous norm but not countably normable [7], gives an example of a locally normable space which is not asymptotically normable (see also Section 2).

B. In this section we shall consider Kothe sequence spaces. Our main aim will be to construct an example of a Kothe space $\lambda(B)$ which is Montel, admits a continuous norm, but still is not asymptotically normable. In particular, $\lambda$ (B) will be an example of a locally normable Kothe space which is not asymptotically normable.

By Proposition 1.3, $\lambda$ (B) cannot be a Schwartz space. In this context we should like to mention that the well-known example constructed by Kothe in [4] of a Montel space which is not 
a Schwartz space, is asymptotically normable by the following proposition which is an immediate consequence of Proposition 1.2 and gives a characterization of asymptotically normable Kothe spaces in terms of the defining matrix.

Proposition 2.1 A Kothe space $\lambda$ (A) is asymptotically normable if and only if there is $\mathrm{k}_{\mathrm{o}}$ such that for every $\mathrm{k}$ we have $\mathrm{p}$ so that for every $\varepsilon>0$ there is an $\mathrm{M}>0$ with

$$
a_{j, k} \leq M a_{j, k_{o}}+\varepsilon a_{j, p^{\prime}} j \in N
$$

Example 2.2 We define

$$
b_{i j, v ; k}= \begin{cases}(i j v)^{k} & \text { for } k \leq v \\ i^{v}(j v)^{k} & \text { for } v<k \leq j+v \\ i_{k-j}(j v)^{k} & \text { for } j+v<k\end{cases}
$$

Then the Kothe space $\lambda(B)$ is Montel, admits a continuous norm but is not asymptotically normable.

Proof.: Suppose for some $\mathrm{k}$ there is a p such that for every $\varepsilon>0$ there is $\mathrm{M}>0$ with

$$
b_{i j, v ; k+1} \leq M b_{i j, v ; k}+\varepsilon b_{i j, v ; p}
$$

for all $(\mathrm{i}, \mathrm{j}, \mathrm{k}) \in \mathrm{N}^{3}$. We may assume $\mathrm{p}>\mathrm{k}+1$, set $\mathrm{v}=\mathrm{k}+1, \mathrm{j}=\mathrm{p}-\mathrm{v}$ and obtain

$$
(i \mathrm{j} \mathrm{v})^{\mathrm{k}+1} \leq \mathrm{M}(\mathrm{i} \mathrm{j} \mathrm{v})^{\mathrm{k}}+\varepsilon \mathrm{i}^{\mathrm{k}+1}(\mathrm{j} \mathrm{v})^{\mathrm{p}}
$$

For $\varepsilon<(j v)^{k+1-p}$, we let $i$ tend to infinity and get a contradiction. Hence the Kothe space $\lambda$ (B) is not asymptotically normable by Proposition 1.2.

Suppose we fix $\mathrm{k}_{\mathrm{o}}$ and let $\mathrm{I} \subset \mathrm{N}^{3}$ be an index set such that for all $\mathrm{k}$ we have

$$
b_{i j v ; k} \leq \rho(k) b_{i j, v ; k}
$$

for some $\rho(k)>0$ and $(i, j, v) \in$ I. If we show that I must be finite, then we have that $\lambda(B)$ is a Montel space [4]. Since the exponent of $i$ is increasing with $k$, we have $j v \leq \rho\left(k_{0}+1\right)$ for all $(i, j$, $v) \in I$. For any given $j_{0}, v_{o}$ let $k>j_{0}+v_{o}+k_{o}$. If $\left(i, j_{o}, v_{0}\right) \in I$, we get

$$
i^{k-j_{o}}\left(j_{o} v_{o}\right)^{k} \leq \rho(k) i^{m}\left(j_{o} v_{o}\right)^{k_{o}}
$$

where $m$ is some integer which is strictly less than $k-j_{o}$ in all cases. Hence $\left\{i:\left(i, j_{0}, v_{o}\right) \in I\right\}$ is finite and so I must also be finite.

C. This section is devoted to the proof of our main theorem 3.3.

For this, we state the following lemma without proof:

Lemma 3.1 Let $A=\left(a_{i, k} ; m\right)$ be a doubly indexed matrix and $A_{k}=\left(a_{i, k ; m}+a_{i+1, k ; m}\right)$. We assume that with suitable sequences $\mathrm{i}(\mathrm{m})$ and $\mathrm{s}(\mathrm{m}) \geq \mathrm{m}$ we have the following:

$$
\begin{aligned}
& \mathrm{a}_{\mathrm{i}, \mathrm{k} ; \mathrm{k}}=1 \\
& \mathrm{a}_{\mathrm{i}, \mathrm{k} ; \mathrm{m}} \geq \mathrm{a}_{\mathrm{i}+1, \mathrm{k} ; \mathrm{m}} \\
& \mathrm{a}_{\mathrm{i}, \mathrm{k} ; \mathrm{m}} \leq \mathrm{a}_{\mathrm{i}+1, \mathrm{k} ; \mathrm{m}} \\
& \mathrm{a}_{\mathrm{i}, \mathrm{k} ; \mathrm{lim}} 0 \\
& \sum_{i} \frac{\dot{d_{i}}}{a_{i, k_{j}}}<+\infty m
\end{aligned}
$$$$
\text { for all } \mathrm{i}, \mathrm{k} \text {. }
$$$$
\text { for all } \mathrm{i}, \mathrm{m} \leq \mathrm{k} \text {. }
$$$$
\text { for all } i \geq i(m), m>k \text {. }
$$$$
\text { for all } \mathrm{m}<\mathrm{k} \text {. }
$$

Then there exists an exact sequence

$$
0 \rightarrow \lambda\left(\mathrm{A}_{\mathrm{K}}\right) \rightarrow \lambda(\mathrm{A}) \rightarrow \omega \rightarrow 0
$$

We will now show that for a given Kothe-Schwartz space $\lambda(B)$ there is another such space $\lambda$ (A) and a surjection of $\lambda$ (A) onto $\omega$ whose kernel is isomorphic to $\lambda$ (B).

Proposition 3.2 For every Kothe-Schwartz space $\lambda$ (B) with a continuous norm, there exists a Kothe-Schwartz space $\lambda(\mathrm{A})$ with a continuous norm and an exact sequence

$$
0 \rightarrow \lambda(\mathrm{B}) \rightarrow \lambda(\mathrm{A}) \rightarrow \omega \rightarrow 0 \text {. }
$$

Further, if $\lambda(\mathrm{B})$ is nuclear, then $\lambda(\mathrm{A})$ is also nuclear. 
Proof: We assume without loss of generally $\mathrm{b}_{\mathrm{j} ; 1}>0$ for all $\mathrm{j}$ and $\lim _{j} \frac{b_{j ; m}}{b_{j ; m+1}}=0$ for all $\mathrm{m}$.

We determine inductively disjoint, infinite sets of integers $M_{k}=\{n(i, k): i=1,2, \ldots\}$ with $\cup \mathrm{M}_{\mathrm{k}}=\mathrm{N}$ such that the following are true:

$$
\begin{array}{ll}
\frac{b_{n(i, k) ; m}}{b_{n(i, k) ; k}} \geq \frac{b_{n(i+1, k) ; m}}{b_{n(i+1, k) ; k}} & \text { for } \mathrm{m}<\mathrm{k} \\
\frac{b_{n(i, k) ; m}}{b_{n(i, k) ; k}} \leq \frac{b_{n(i+l, k) ; m}}{b_{n(i+1, k) ; k}} & \text { for } \mathrm{m}>\mathrm{k}, \mathrm{i} \geq \mathrm{m} \\
\frac{b_{n(i, k) ; m}}{b_{n(i, k) ; m+1}} \leq 2^{-i} & \text { for } \mathrm{m} \leq \mathrm{i} .
\end{array}
$$

For a fixed $\mathrm{k}$, the sequence $(\mathrm{n}(\mathrm{i}, \mathrm{k}))_{\mathrm{i}=1,2, \ldots . . .}$ can be chosen inductively, starting with $\mathrm{n}(1, \mathrm{k})$ $=\min \left\{\mathrm{N} \backslash \bigcup_{l=l}^{k-l} M_{l}\right\}$ and such that $\left\{\mathrm{N} \backslash \bigcup_{l=l}^{k} M_{l}\right\}$ remains infinite. We now set

$$
\mathrm{a}_{\mathrm{i}, \mathrm{k} ; \mathrm{m}}= \begin{cases}\frac{b_{n(i, k) ; m}}{b_{n(i, k) ; k}} & \text { for } \mathrm{m}<\mathrm{k} \\ \frac{b_{n(i-1, k) ; m}}{b_{n(i-1, k) ; k}} & \text { for } \mathrm{m}>\mathrm{k}, \mathrm{i} \geq 2 \\ 1 & \text { elsewhere }\end{cases}
$$

Since $a_{i, k ; k}=a_{i+1, k ; k}=1$, we note that $\left(a_{i, k ; m}\right)$ is increasing in $m$. Then (1) ..... (4) of 3.1 Lemma are satisfied and hence we have an exact sequence $\quad 0 \rightarrow \lambda\left(\mathrm{A}_{\mathrm{K}}\right) \rightarrow \lambda(\mathrm{A}) \rightarrow \omega \rightarrow 0$. One can check easily that $\lambda(\mathrm{A})$ is a Schwartz space or if $\lambda(\mathrm{B})$ is nuclear, then $\lambda(\mathrm{A})$ is also nuclear.

For $\mathrm{m}<\mathrm{k}$ and $\mathrm{m} \geq \mathrm{k}$ using (5) and (6) are estimate in different ways and obtain

$$
\frac{b_{n(i, k) ; m}}{b_{n(i, k) ; k}} \leq a_{i, k ; m}+a_{i+1, k ; m} \leq 2 \frac{b_{n(i, k) ; m}}{b_{n(i, k) ; k}}
$$

for $\mathrm{i}>\mathrm{m}$. Therefore $\lambda\left(\mathrm{A}_{\mathrm{K}}\right)$ is isomorphic to $\lambda(\mathrm{B})$ by diagonal transformation.

Theorem 3.3 A Frechet space $E$ is asymptotically normable if and ony if there is an index set I and a nuclear Kothe space $\lambda(\mathrm{A})$ with a continuous norm such that $\mathrm{E}$ is isomorphic to a subspace of $\mathrm{L}_{\infty}(\mathrm{I}) \hat{\otimes}_{\pi} \lambda(\mathrm{A})$.

Proof: We have a nuclear Kothe space $\lambda(\mathrm{B})$ with a continuous norm such that $\quad(\mathrm{E}, \lambda(\mathrm{B})) \in$ $\left(S_{l}^{*}\right)$ by Lemma 5.4 in [3]. By the previous result we construct an exact sequence.

$$
0 \rightarrow \lambda(\mathrm{B}) \rightarrow \lambda(\mathrm{A}) \rightarrow \omega \rightarrow 0 \text {. }
$$

If $\mathrm{E}$ is a Banach space, our theorem is trivial. If $\mathrm{E}$ is a proper Frechet space, then (E, $\lambda(B)) \in\left(S_{1}^{*}\right)_{0}$ by Lemma 3.3 in [5]. Now we choose a set I such that each Banach space $\mathrm{E}_{\mathrm{k}}$ is isomorphic to a subspace of $\mathrm{L}_{\infty}(\mathrm{I})$, where $\mathrm{E}_{\mathrm{k}}$ is the completion of $\left(\mathrm{E},\|\|_{\mathrm{k}}\right)$. We identify the tensor product $\mathrm{L}_{\infty}(\mathrm{I}) \otimes_{\pi} \lambda(B)$ with the space of all $\mathrm{y}=\left(\mathrm{y}_{\mathrm{j}}\right)$, such each $\mathrm{v}_{\mathrm{j}} \in \mathrm{L}_{\infty}(\mathrm{I})$ and for every $\mathrm{k} \in \mathrm{N}$ we have $\quad\|y\|_{k}=\sup _{j}\left\|y_{j}\right\|_{\infty} b_{j ; k}<+\infty$

where \|\|$_{\infty}$ denotes the norm of $\mathrm{L}_{\infty}(\mathrm{I})$. An obvious modification of Proposition 3.5 in [5] yields $\left(E, L_{\infty}(I) \hat{\otimes}_{\pi} \lambda(B) \in\left(S_{1}\right)\right.$. Taking tensor product with $\mathrm{L}_{\infty}(\mathrm{I})$ we get an exact sequence 


$$
0 \rightarrow \mathrm{L}_{\infty}(\mathrm{I}) \hat{\otimes}_{\pi} \lambda(\mathrm{B}) \rightarrow \mathrm{L}_{\infty}(\mathrm{I}) \hat{\otimes}_{\pi} \lambda(\mathrm{A}) \rightarrow \mathrm{L}_{\infty}(\mathrm{I})^{\mathrm{N}} \rightarrow 0
$$

Since Ext $\left(E, L_{\infty}(I) \hat{\otimes}_{\pi} \lambda(B)\right)=0$, the natural imbedding $\bar{T}$ of $E$ into $L_{\infty}(I)^{N}$ can be lifted to a continuous linear operator $\mathrm{T}: \mathrm{E} \rightarrow \mathrm{L}_{\infty}(\mathrm{I}) \hat{\otimes}_{\pi} \lambda(\mathrm{A})$. It is easily checked that $\overline{\mathrm{T}}$ is one-to-one and has a closed range.

We would like to point out that the Kothe space $\lambda(\mathrm{A})$ we have constructed actually depends only on $\varphi$. This is so, because for each strictly increasing function $\varphi:(0, \infty) \rightarrow(0, \infty)$, an examination of the proof of Lemma 5.4 in [3] reveals that there is a nuclear Kothe space $\lambda(B)$ such that $(\mathrm{E}, \lambda(\mathrm{B})) \in\left(S_{I}^{*}\right)$ for any Frechet space $\mathrm{E}$ which satisfies $\left(\mathrm{DN}_{\varphi}\right)$.

Finally, we would like to note that L. Holmstrom [8] proved that every nuclear asymptotically normable Frechet space is isomorphic to a subspace of some nuclear Kothe space which admits a continuous norm.

D. Finally we use the results of section 3 for another description of the class of asymptotically normable Frechet spaces and for a comparison with the class quasi-normable Frechet spaces. The first Lemma is obvious.

Lemma 4.1 If $\mathrm{E}$ is asymptotically normable and $\mathrm{H} \subset \mathrm{E}$ a subspace then $\mathrm{H}$ is asymptotically normable.

Lemma 4.2 If $\mathrm{E}$ and $\mathrm{F}$ are asymptotically normable then so is $L_{b}\left(E_{b}^{l}, F\right)$.

Proof: The proof is straightforward if one notices that $\mathrm{E}$ being asymptotically normable is equivalent to: there is $\mathrm{k}_{\mathrm{o}}$ such that for every $\mathrm{k}$ there is a p so that for every $\varepsilon>0$ we can choose $\mathrm{M}>0$ with

$$
U_{k}^{o} \subset M U_{k_{o}}^{o}+\varepsilon U_{p}^{o} \quad \text { From these Lemmas and Theorem 3.3, we can obtain: }
$$

Theorem 4.3 The class of asymptotically normable Frechet spaces is the smallest class of Frechet spaces containing the nuclear Kothe spaces with continuous norm the Banach spaces and is closed under $\varepsilon$-tensor products and subspaces.

For comparison we recall that the class of quasi-normable Frechet spaces is the smallest class of Frechet spaces containing the nuclear Kothe spaces (or nuclear Kothe spaces with continuous norms, or Schwartz spaces, or countably normable Schwartz spaces) and the Banach spaces and is closed under $\pi$-tensor products and quotient spaces.

\section{References}

[1] A. Grothendieck, Topological vector spaces, New York - London-Paris, (1973).

[2] R. Meise, D. Vogt, Math. Nachr., 122 (1985) 141.

[3] D. Vogt, Some results on continuous linear maps between Frechet spaces, in Bierstedt K.D., Fuchssteiner B. (eds.), <<Functional Analysis : Surveys and Recent Results II $>>$, North-Holland Math. Studies, 90 (1984), pp. 349-381.

[4] G. Kothe, Topological vector spaces, I, II, New York-Heildelber-Berlin, 3 (1969, 1979).

[5] D. Vogt, Studia Math., 85 (1987) 163.

[6] T. Terzioglu, D. Vogt, Math., 54 (1990)180.

[7] E. Dubinsky, Studia Math., 71 (1981) 85.

[8] L. holmstrom, Proc. Amer. Math. Soc., 89 (1983)453.

\section{Further reading}

[1] E. Dubinsky, The structure of nuclear Frechet spaces, Lecture notes in Math., 720 (1979), Berlin-Heildel-berg-New York.

[3] E. Dubinsky, D. Vogt, Frechet spaces with quotients failing the bounded approximation property, Studia Math., 81 (1985), pp.71-77.

[11] G.L., Litvinov, Nuclear space, Springer Verlag, New York, (2001). 